\title{
Dew Point Time Series Forecasting at the North Dakota
}

\author{
Bugrayhan Bickici Arikan 1,", Luo Jiechen ${ }^{2}$, Ibrahim I D Sabbah ${ }^{3}$, Ahmed Ewees ${ }^{4,5}$, Rajab Homsi ${ }^{6}$, \\ Sadeq Oleiwi Sulaiman ${ }^{7,8}$ \\ ${ }^{1}$ Department of Civil Engineering, Istanbul Medeniyet University, Istanbul, Turkey; \\ bugrayhan.bickici@medeniyet.edu.tr \\ ${ }^{2}$ School of Computer Science, Baoji University of Arts and Sciences, China; superluojiechen@163.com \\ ${ }^{3}$ Modeling Evolutionary Algorithms Simulation and Artificial Intelligence, Faculty of Electrical \& Electronics \\ Engineering, Ton Duc Thang University, Ho Chi Minh City, Viet Nam; Ibrahemsabbah1995@gmail.com \\ ${ }^{4}$ Department of e-Systems, University of Bisha, Bisha 61922, Saudi Arabia; a.ewees@ hotmail.com \\ ${ }^{5}$ Department of Computer, Damietta University, Damietta 34517, Egypt \\ ${ }^{6}$ Department of Water \& Environmental Engineering, Faculty of Civil Engineering, University of Aleppo, Syria; \\ eng.rajabhomsi@gmail.com \\ ${ }^{7}$ Dams and Water Resources Department, College of Engineering, University of Anbar, Ramadi, Iraq; \\ sadeq.sulaiman@uoanbar.edu.iq \\ ${ }^{8}$ Civil, Environmental and Natural Resources Engineering, Lulea University of Technology, Lulea, Sweden \\ * Correspondence: bugrayhan.bickici@medeniyet.edu.tr
}

Manuscript received: 23-07-2021, revised: 21-08-2021, accepted: 26-08-2021.

\begin{abstract}
Hydrological time series forecasting is one of the hot topics in the domain of statistical hydrology. Providing accurate forecasting can contribute to diverse applications for catchment sustainability and management. Dew point temperature $\left(T_{d e w}\right)$ is one of the complex hydrological processes that highly essential to be quantified accurately for several catchment activities such as crops, agriculture, and others. In this study, three types of models' recursive strategy, direct strategy, and DirRec which is the combination of recursive and direct strategies were adopted to obtain $h$-steps ahead predictions of $T_{d e w}$. Ten years monthly scale dataset of $T_{d e w}$ at two meteorological stations (Beach and Cavalier) located at the North Dakota, USA, were used for the modeling development. The performance of the considered models was compared with two benchmark models: autoregressive moving average (ARIMA) and exponential smoothing (ETS). Modeling results indicated that, compared with the benchmark models, the proposed methods gave good results for the multiahead forecasting. For instance, for Cavalier station, the root mean squared prediction errors obtained from the proposed and benchmark methods when the forecast horizon is 12 are as follows: recursive strategy $(\mathrm{RMSPE}=3.731)$ direct strategy $(\mathrm{RMSPE}=3.385)$, DirRec $(\mathrm{RMSPE}=3.141)$, ARIMA $(\mathrm{RMSPE}=12.957)$, and ETS (RMSPE $=27.479)$.
\end{abstract}

Keywords: Dew point temperature; North Dakota; $h$-steps ahead predictions.

\section{Introduction}

Hydrological cycle processes modeling is one of the essential concern for hydrologists [1], [2]. This is due to the actual association of the high complexities of the nature pattern [3]. In various fields, such as hydrology, agriculture, agronomy, and climatology, dew point temperature $\left(T_{\text {dew }}\right)$ estimation is considered highly important for its role in the calculation of the relative humidity, actual vapour pressure, and because of its importance for plants survival in areas with low precipitation [4]. In addition, dew point presented a highly significant hydrological process that contribute to several water resource planning, management and sustainability [5], [6]. Further, there are several watershed hydrological engineering practice, which require precise quantification for the dew point temperature [7]. Moreover, dew point temperature is an important parameter for the prediction of long-term changes in climate [8]. Over the last few decades, artificial intelligence ( $\mathrm{AI}$ ) models have found vast application in the modeling and estimation of various engineering and sciences problems [9]-[12]. However, a remarkable advancement has been observed within the natural process applications such as hydrology [13], [14], climate [15], morphologies [16], [17] and environnement [18], [19].

Numerous soft computing models have recently been developed for $T_{\text {dew }}$ estimation [20], [21]; for instance, the use of artificial neural networks (ANN) model for 1 to $12 \mathrm{~h}$ ahead $T_{\text {dew }}$ prediction based on several input parameters (such as relative humidity, air temperature, vapor pressure, solar radiation, wind speed, rainfall, etc.) has been reported [22]. The outcome of the study showed that ANN model succeeded in 
achieving accurate $T_{\text {dew }}$ prediction in the state of Georgia, USA. Furthermore, the study by [23] reported the use of ensemble ANN models prediction of up to $12 \mathrm{~h} T_{\text {dew }}$. The study relied on Fuzzy membership functions (in consideration of the day of the year) for the identification of the weights of the developed ANN structure. ANN model has been used with the Levenberg-Marquardt algorithm by [24] for the prediction of hourly $T_{d e w}$; the performance of both models was compared with that of a multiple linear regression (MLR) model and from the results, the use of wind vector, climate variables and weather conditions as inputs was found to slightly improve the accuracy of both MLR and ANN models. However, the ANN and Levenberg-Marquardt algorithm performed significantly better than the MLR model. The use of two ANNs (i.e., generalized regression neural networks, GRNN, and Kohonen self-organizing feature maps, KSOFM) and two ANFIS models (i.e. ANFIS with sub-clustering, ANFIS-SC, and ANFIS with grid partitioning, ANFIS-GP) for daily $T_{\text {dew }}$ prediction at 3 stations in the Korean Republic has been reported [8]. During the study, different combinations of air temperature, sunshine hours, relative humidity, wind speed, and saturation vapour pressure were used as inputs and from the results, the performance of the models was similar except for the KSOFM model, which performed the least. The performance of ANN and gene expression programming (GEP) models in daily $T_{\text {dew }}$ prediction has been compared in the Korean republic using different climatic parameters [25]. The outcome of the study showed that the GEP model achieved a higher level of performance than the ANN model in daily $T_{d e w}$ prediction.

Extreme learning machine (ELM) model has been reportedly used in daily $T_{\text {dew }}$ prediction at two stations in Iran; the performance of the ELM model was compared with those of ANN and SVR models and the ELM model was found to achieve better performance than the other two models in terms of achieving accurate prediction [26]. Another study in the USA relied on ANN and MLR for daily average $T_{d e w}$ prediction in southwest Georgia (USA) [27]. Due to the lack of metrological variables, only temperature and precipitation were used in the study as inputs and the results showed that the ANN model performed reasonably better than the MLR model. However, the error levels of both models for the colder $T_{\text {dew }}$ were high due to data unavailability. The performance of GEP, the M5 model tree, and SVR in the estimation of daily $T_{\text {dew }}$ of Tabriz, Iran using different climatic parameters has been evaluated [28]. Notably, the M5 model tree model outperformed the rest of the models in achieving better prediction performance. SVR and ELM were used by [14] for daily $T_{\text {dew }}$ prediction in the humid and semi-arid regions of India. The input variables used for both models were relative humidity, wet bulb temperature and vapour pressure and from the results, the performance of the ELM model was superior to that of the SVR model. Also, recently there have been several investigation on the implementation on advance machine learning approaches for the average dew point temperature prediction [29], [30].

Several other AI implementations have been observed for the $T_{\text {dew }}$ simulation such as hybridized AI models with nature inspired optimization algorithms [4], the capacity of some new data mining [7]. Although, there have several investigations for $T_{\text {dew }}$ using different AI models. There is still several drawbacks have been approved with the development of classical version of AI models such as ANN, SVR, ANFIS models such as the overfitting, learning process limitations, hyperparameters tuning [31]-[34]. Hence, the exploration of new version of robust soft computing technologies to overcome the mentioned limitations is always the motive for engineers and decision makers [35]-[37]. In addition, based on the researches discussed above, the main aim generally has been paid to forecasting one-step ahead prediction of $T_{\text {dew }}$. In many scientific fiels including meteorology and hydrology, the one-step ahead forecast may not be informative because the decision makers may need long-term forecasts to manage risks properly. Thus, h-steps ahead $(\mathrm{h}>1)$ prediction of $\mathrm{T}_{\mathrm{dew}}$ may be more helpful for decision makers compared with one-step ahead prediction since the former allows to make a long-term plans easily.

From the above-discussed arguments, the main goal of this paper is to introduce three strategic based on $h$-steps ahead prediction techniques called recursive strategy, direct strategy, and DirRec strategy which is the combination of recursive and direct strategies to effectively obtain $h$-steps ahead predictions of $T_{d e w}$. A real-observation of $T_{d e w}$ from two stations is used for building and testing the proposed AI-models that the case studies consist of one-month interval for a ten-year period. The prediction performance of the considered $h$-steps ahead prediction techniques are compared with favourably existing methods via several error metrics.

\section{Materials and Methods}

\subsection{Case study}

In the current research, two meteorological stations (Beach and Cavalier) located at the north Dakota were used for the modeling development. Time series of $T_{d e w}$ for 10 years' time period (January, 2010 - December, 2019). The time series plots of the observed $T_{d e w}$ are presented in Figure 1 . In addition, the summary statistics for the observed $T_{d e w}$ for each station are reported in Table 1 . The Case study and meteorological stations location presented in Figure 2. 

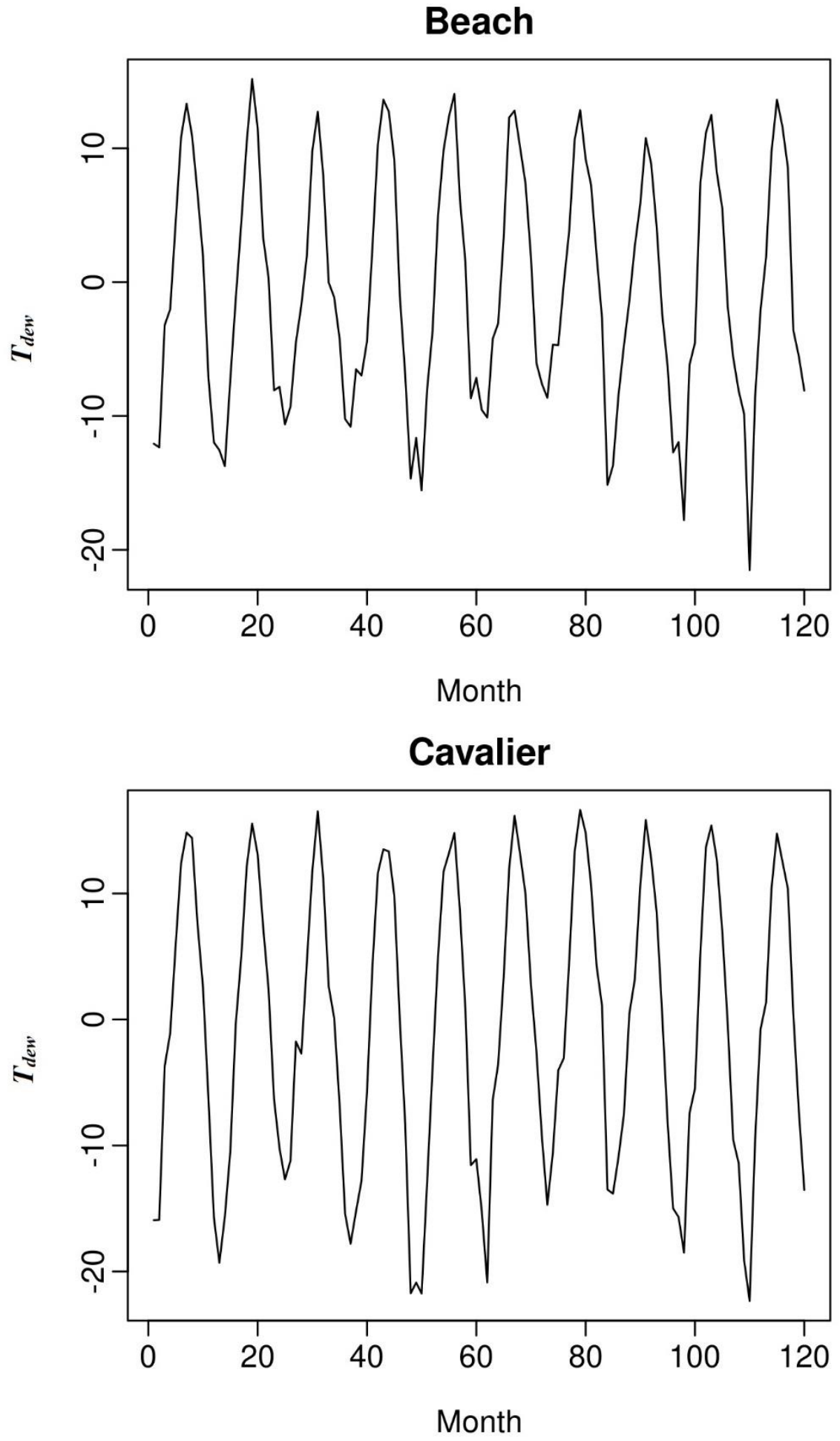

Figure 1. Time series plots of monthly $T_{\text {dew }}$ values during 2010-2020; Beach station (upper panel) and Cavalier station (lower panel).

Table 1: Summary statistics of monthly $T_{d e w}$ values during 2010-2020.

\begin{tabular}{ccccccc}
\hline Station & Min & Mean & Max & STD & Skewness & Kurtosis \\
\hline Beach & -21.5170 & -0.4176 & 15.1690 & 8.9011 & 0.0120 & 1.9180 \\
Cavalier & -22.3740 & -0.7563 & 16.6320 & 11.3934 & -0.1365 & 1.7736 \\
\hline
\end{tabular}



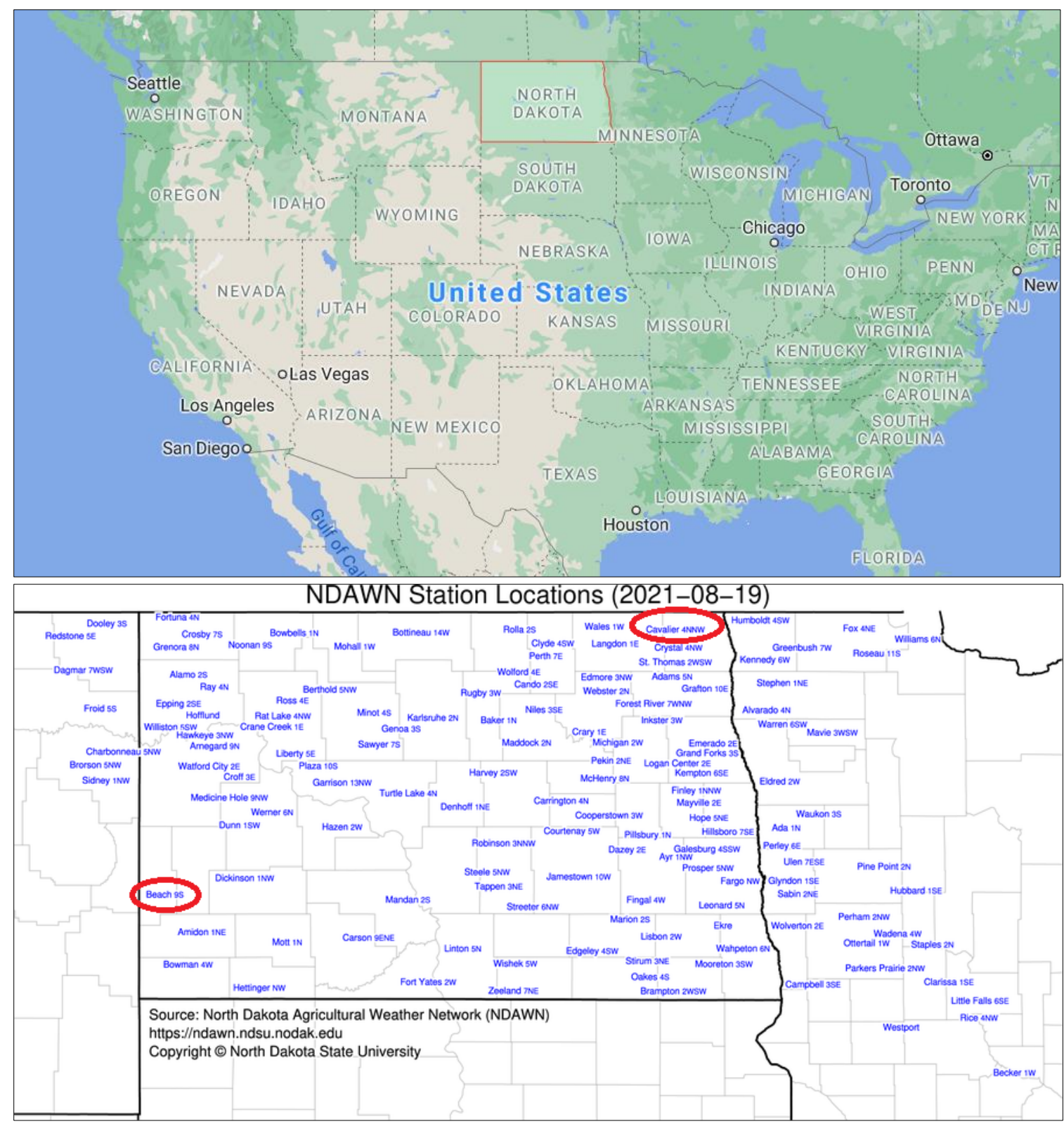

Figure 2. The location of the selected meteorological stations at North Dakota.

\subsection{Applied methods explanation}

This section is devoted to present the details of the $h$-steps ahead prediction techniques. Let $\left\{y_{1}, y_{2}, \ldots, y_{n}\right\}$ denote a realization of the stochastic process $y$. It is of a great interest to predict a future realization of this process consisting of $h=1,2, \ldots, H$, where $h$ denotes the forecasting horizon, values conditionally on $\left\{y_{1}, y_{2}, \ldots, y_{n}\right\}$, i.e., $\left\{y_{n+1}, y_{n+2}, \ldots, y_{n+H}\right\}$. In this context, the first strategy called recursive strategy repeats several one-steps ahead prediction procedures until all the predictions in the forecast horizon are obtained. In more detail, let $f$ and $e$ respectively denote the functional dependency between the past observations and modelling error. Then, a one-step ahead prediction is first obtained based on the functional dependency between the past observations as follows:

$$
y_{t+1}=f\left(y_{t}, y_{t-1}, \ldots, y_{t-d+1}\right)+e
$$

where $t \in\{d, \ldots, n-1\}$ denotes the embedding dimension (see e.g., [38], [39]). Let $f$ denote the trained model obtained by one-step ahead prediction. Then, the $H$-steps ahead predictions are given by:

$$
\hat{y}_{n+h}=\left\{\begin{array}{c}
\hat{f}\left(y_{n}, y_{n-1}, \ldots, y_{n-d+1}\right), \text { if } h=1, \\
f\left(\hat{y}_{n-h-1}, \ldots, \hat{y}_{n+1}, y_{n}, \ldots, y_{n-d+h}\right), \text { if } h \in\{2, \ldots, d\} \\
\hat{f}\left(\hat{y}_{n+h-1}, \ldots, \hat{y}_{n+h-d}\right), \text { if } h \in\{d+1, \ldots, H\} .
\end{array}\right.
$$


The workflows of one-step ahead and h-steps ahead prediction are presented in Figure 3. From this figure, the h-steps ahead prediction procedure can be considered as a repeated case of one-step ahead prediction process.

A)

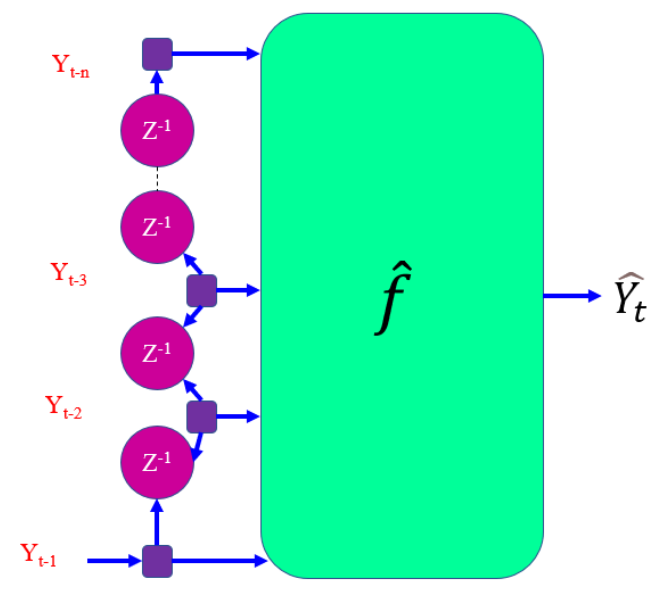

B)

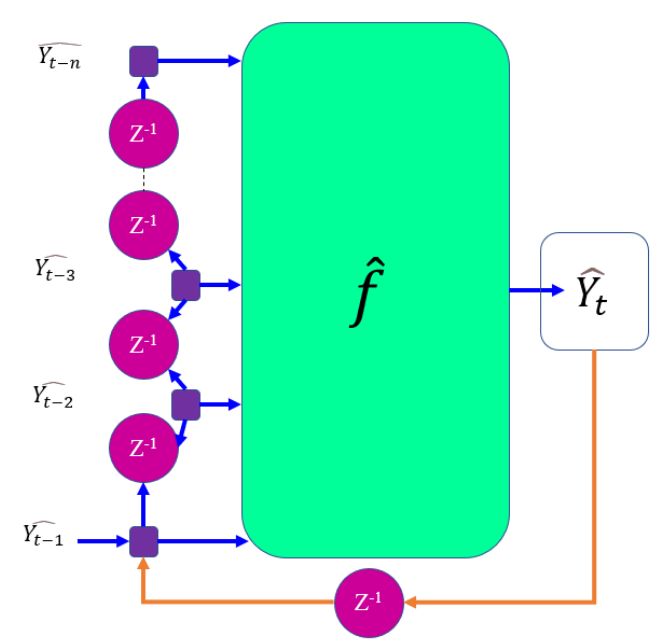

Figure 3: Workflow of one-step-ahead (A)) and $h$-steps-ahead (B)) forecasting. The trained model returns the predicted value of time series at step $t+1$. $\mathrm{z}^{-1}$ denotes the unit delay operator, i.e., $y_{t-1}=z^{-1} y_{t}$.

In recursive strategy, the computed predictions may deviate greatly from their true values when the forecasting horizon is greater than the embedding dimension, i.e., $h>d$. This is due to fact that the recursive strategy is sensitive to the accumulation of errors with $h$ [39].

The direct strategy, contrary to recursive strategy, applied $H$ independent models in each forecasting horizon to obtain $H$-steps ahead predictions. Let $f_{h}$ denote the trained model at step $h$. Then, the realization at this step is given by:

$$
y_{t+h}=f_{h}\left(y_{t}, y_{t-1}, \ldots, y_{t-d+1}\right)+e
$$

where $t \in\{d, \ldots, n-H\}$ and $h \in\{1,2, \ldots H\}$. The forecasts at step $h$ are obtained via the learned models $f_{h}$ are then obtained as follows:

$$
\hat{y}_{n+h}=\hat{f}_{n}\left(y_{n}, y_{n-1}, \ldots, y_{n-d+1}\right)
$$

In other words, this strategy uses directly the observed values, rather than approximates values, to obtain predictions, which results in reducing accumulation of errors. On the other hand, it may have lack of forecasting accuracy since the direct strategy independently obtains the forecasts since the dependencies between the observations are ignored in the prediction process.

The DirRec strategy proposed by [40] computes the predictions by combining recursive and direct strategies. In more detail, similar to the direct strategy, the DirRec strategy computes the predictions by constructing different models in each forecast horizon but enlarges the set of inputs by adding variables corresponding to the forecasts of previous step as in recursive strategy [39]. Independently from the previous two strategies, the DirRec strategy uses different embedding size for all horizons. Let $f_{h}$ denote model at horizon $h$, then, the true realization of future observations are defined by:

$$
y_{t+h}=f_{h}\left(y_{t+h-1}, \ldots, y_{t-d+1}\right)+e
$$

with $t \in\{d, \ldots, n-H\}$ and $h \in\{1, \ldots, H\}$. Then, the DirRec strategy computes the forecasts using $h$ learned models as follows:

$$
\hat{y}_{n+h}=\left\{\begin{array}{c}
f_{h}\left(y_{n}, \ldots, y_{n-d+1}\right), \text { if } h=1, \\
f_{h}\left(\hat{y}_{n+h-1}, \ldots, \hat{y}_{n+1}, y_{n}, \ldots, y_{n-d+1}\right), \text { if } h \in\{2, \ldots, H\}
\end{array}\right.
$$

As showed by [39], [41], this strategy outperforms direct and to recursive strategies.

In this study, the neural network (NN) machine learning algorithm with one-hidden layer is used with the direct and recursive strategies to obtain $h$-steps ahead predictions. Let $m(y)$ denote the model component, the considered $\mathrm{NN}$ algorithm is given by:

$$
m(y)=b_{0}+\sum_{j=1}^{J} b_{j} g\left(w_{j}^{T} y\right)
$$


where $w_{j}$ is the vector of weights associated with $j^{t h}$ hidden node, $b_{0}, b_{1}, \ldots, b_{n}$ are the weights for the output note and, $J$ denotes the number of hidden nodes. Herein, the logistic function $g(a)=1 /\left(1+e^{a}\right)$ is considered. On the other hand, for the DirRec strategy, the linear model:

$$
m(y)=\sum_{j=1}^{p} \beta_{j} y_{j}
$$

where $\beta_{j}$ denotes the model parameter for $j^{\text {th }}$ input variable, the gradient boosting algorithm proposed by [40], with univariate P-splines are considered to obtain predictions.

\section{Application results and assessment}

The prediction performance of multi-steps ahead prediction methods are evaluated using ten years of monthly data. The performances of the methods were compared with three existing traditional prediction methods: autoregressive moving average (ARIMA) and exponential smoothing (ETS). The results for the benchmark methods are obtained using the $R$ package "forecast" [42]. The out-of-sample prediction performance of the methods are compared with three error metrics: root mean square prediction error (RMSPE), mean absolute prediction error (MAPE), and average absolute percent relative prediction error (AAPRPE) as follows:

$$
\begin{aligned}
R M S P E & =\sqrt{\frac{1}{H} \sum_{h=1}^{H}\left(y_{n+h}-\hat{y}_{n+h}\right)^{2}} \\
M A P E & =\frac{1}{H} \sum_{h=1}^{H}\left|y_{n+h}-\hat{y}_{n+h}\right| \\
\text { AAPRPE } & =\frac{100}{H} \sum_{h=1}^{H}\left(\left|\frac{y_{n+h}-\hat{y}_{n+h}}{y_{n+h}}\right|\right)
\end{aligned}
$$

With all the considered methods, the first eight years (2010-2017) are used to construct model. Then, $H$ $=[1,6,12]$-steps ahead predictions are computed based on the constructed models and all the three-error metrics (e.g., RMSPE, MAPE, and AAPRPE) are computed. While doing so, first, the 96 observations in the dataset (eight years of data), called training data, are used to construct models and to predict $H=[1,6,12]$ steps ahead observations. Then, the training sample is increased by one month and the next $H=[1,6,12]$ steps ahead observations are predicted. This process is repeated until all the observations in the forecast horizon predicted. In addition to the computed error metrics, the standard deviation for each forecast horizons are computed to compare the stability of the methods.

The results are presented in Tables 2 and 3. From these tables, it is obvious that machine learning based $h$-steps ahead methods produce improved prediction performance compared with ARIMA and ETS. In addition, the results have shown that all the machine learning based methods produce similar performance for short term prediction (i.e., $h=1)$. In this case, the recursive and direct strategies produce better performance than the DirRec strategy. For moderate forecast horizon (i.e., $h=6$ ), the direct strategy produces improved performance than all the methods. On the other hand, for long-term forecast (i.e., $h=12$ ), the DirRect strategy produces the best performance among others. Tables 2 and 3 also present the standard error values of the computed error metrics for all forecast horizons and methods. From the results, it is clear that the DirRect strategy generally produces smaller standard errors compared with other alternatives. This result indicates that the DirRect strategy produces more stable results among others. All in all, our results demonstrate that, the direct strategy seems a better prediction method for short-term and moderate-term predictions while the DirRec strategy is more suitable one among others for long-term predictions.

Table 2. Computed performance metrics for Beach station with standard deviations given in bracket.

\begin{tabular}{|l|l|l|l|l|}
\hline Metric & Method & \multicolumn{3}{|c|}{ Forecast horizon } \\
\hline \multirow{4}{*}{ RMSPE } & & $\mathrm{H}=1$ & $\mathrm{H}=6$ & $\mathrm{H}=12$ \\
& Direct & 3.029 & $\mathbf{3 . 8 2 1}$ & 4.928 \\
& & $(2.805)$ & $\mathbf{( 1 . 2 6 5 )}$ & $(1.150)$ \\
\cline { 2 - 5 } & Recursive & $\mathbf{2 . 9 3 9}$ & 4.174 & 4.064 \\
& & $\mathbf{( 2 . 5 0 6 )}$ & $(1.281)$ & $(0.697)$ \\
\cline { 2 - 5 } & DirRec & 3.019 & 3.870 & $\mathbf{4 . 0 0 5}$ \\
& & $(2.888)$ & $(1.414)$ & $\mathbf{( 0 . 5 8 6 )}$ \\
\cline { 2 - 5 } & ARIMA & 5.107 & 10.818 & 10.960 \\
& & $(3.683)$ & $(3.160)$ & $(1.174)$ \\
\hline
\end{tabular}




\begin{tabular}{|c|c|c|c|c|}
\hline & ETS & $\begin{array}{l}5.276 \\
(4.748)\end{array}$ & $\begin{array}{l}18.225 \\
(12.011)\end{array}$ & $\begin{array}{l}27.400 \\
(18.486)\end{array}$ \\
\hline \multirow{5}{*}{ MAPE } & Direct & $\begin{array}{l}3.029 \\
\mathbf{( 2 . 5 0 6 )}\end{array}$ & $\begin{array}{l}\mathbf{2 . 9 3 8} \\
(0.818) \\
\end{array}$ & $\begin{array}{l}3.725 \\
(1.142)\end{array}$ \\
\hline & Recursive & $\begin{array}{l}\mathbf{2 . 9 3 9} \\
(2.805) \\
\end{array}$ & $\begin{array}{l}3.117 \\
\mathbf{( 0 . 7 6 9 )} \\
\end{array}$ & $\begin{array}{l}3.150 \\
(0.740) \\
\end{array}$ \\
\hline & DirRec & $\begin{array}{l}3.019 \\
(2.888)\end{array}$ & $\begin{array}{l}3.024 \\
(0.925)\end{array}$ & $\begin{array}{l}3.065 \\
(0.515)\end{array}$ \\
\hline & ARIMA & $\begin{array}{l}5.107 \\
(3.683)\end{array}$ & $\begin{array}{l}9.560 \\
(3.244)\end{array}$ & $\begin{array}{l}8.704 \\
(1.168)\end{array}$ \\
\hline & ETS & $\begin{array}{l}5.276 \\
(4.748) \\
\end{array}$ & $\begin{array}{l}15.670 \\
(11.211)\end{array}$ & $\begin{array}{l}23.692 \\
(16.304)\end{array}$ \\
\hline \multirow{5}{*}{ AAPRPE } & Direct & $\begin{array}{l}50.889 \\
(60.966)\end{array}$ & $\begin{array}{l}\mathbf{7 . 4 5 4} \\
(2.373) \\
\end{array}$ & $\begin{array}{l}5.527 \\
(2.063) \\
\end{array}$ \\
\hline & Recursive & $\begin{array}{l}51.124 \\
(62.083) \\
\end{array}$ & $\begin{array}{l}8.065 \\
\mathbf{( 2 . 2 0 8 )} \\
\end{array}$ & $\begin{array}{l}4.630 \\
(1.685) \\
\end{array}$ \\
\hline & DirRec & $\begin{array}{l}43.902 \\
(43.968)\end{array}$ & $\begin{array}{l}8.197 \\
(2.508)\end{array}$ & $\begin{array}{l}4.134 \\
(0.874)\end{array}$ \\
\hline & ARIMA & $\begin{array}{l}88.598 \\
(94.724)\end{array}$ & $\begin{array}{l}23.266 \\
(11.050)\end{array}$ & $\begin{array}{l}9.239 \\
(1.688)\end{array}$ \\
\hline & ETS & $\begin{array}{l}81.320 \\
(85.377)\end{array}$ & $\begin{array}{l}51.539 \\
(41.870)\end{array}$ & $\begin{array}{l}38.661 \\
(36.703)\end{array}$ \\
\hline
\end{tabular}

Table 3. Computed performance metrics for Cavalier station with standard deviations given in bracket.

\begin{tabular}{|c|c|c|c|c|}
\hline \multirow[t]{2}{*}{ Metric } & \multirow[t]{2}{*}{ Method } & \multicolumn{3}{|c|}{ Forecast horizon } \\
\hline & & $\mathrm{H}=1$ & $H=6$ & $\mathrm{H}=12$ \\
\hline \multirow{5}{*}{ RMSPE } & Direct & $\begin{array}{c}\mathbf{2 . 4 2 5} \\
(2.117) \\
\end{array}$ & $\begin{array}{c}\mathbf{3 . 0 4 4} \\
(1.171) \\
\end{array}$ & $\begin{array}{c}3.731 \\
(1.418) \\
\end{array}$ \\
\hline & Recursive & $\begin{array}{c}3.022 \\
(2.429)\end{array}$ & $\begin{array}{c}3.497 \\
(1.155) \\
\end{array}$ & $\begin{array}{c}3.385 \\
(0.625) \\
\end{array}$ \\
\hline & DirRec & $\begin{array}{c}2.605 \\
\mathbf{( 1 . 8 4 8 )} \\
\end{array}$ & $\begin{array}{c}3.120 \\
(\mathbf{0 . 8 4 9}) \\
\end{array}$ & $\begin{array}{c}3.141 \\
(0.465) \\
\end{array}$ \\
\hline & ARIMA & $\begin{array}{c}5.709 \\
(3.379) \\
\end{array}$ & $\begin{array}{l}13.313 \\
(3.573)\end{array}$ & $\begin{array}{l}12.957 \\
(1.359)\end{array}$ \\
\hline & ETS & $\begin{array}{c}4.730 \\
(3.697) \\
\end{array}$ & $\begin{array}{c}20.989 \\
(10.416)\end{array}$ & $\begin{array}{l}27.479 \\
(9.226) \\
\end{array}$ \\
\hline \multirow{5}{*}{ MAPE } & Direct & $\begin{array}{c}2.425 \\
(2.117) \\
\end{array}$ & $\begin{array}{c}2.559 \\
(1.052) \\
\end{array}$ & $\begin{array}{c}3.047 \\
(1.289) \\
\end{array}$ \\
\hline & Recursive & $\begin{array}{c}3.022 \\
(2.429)\end{array}$ & $\begin{array}{c}2.903 \\
(0.999)\end{array}$ & $\begin{array}{c}2.726 \\
(0.534)\end{array}$ \\
\hline & DirRec & $\begin{array}{c}2.605 \\
\mathbf{( 1 . 8 4 8 )} \\
\end{array}$ & $\begin{array}{c}2.594 \\
(\mathbf{0 . 6 3 1}) \\
\end{array}$ & $\begin{array}{c}2.427 \\
(0.347) \\
\end{array}$ \\
\hline & ARIMA & $\begin{array}{c}5.709 \\
(3.379) \\
\end{array}$ & $\begin{array}{r}11.827 \\
(3.614) \\
\end{array}$ & $\begin{array}{r}11.028 \\
(1.328) \\
\end{array}$ \\
\hline & ETS & $\begin{array}{c}4.730 \\
(3.697) \\
\end{array}$ & $\begin{array}{r}17.616 \\
(9.607) \\
\end{array}$ & $\begin{array}{l}23.840 \\
(8.640) \\
\end{array}$ \\
\hline \multirow{5}{*}{ AAPRPE } & Direct & $\begin{array}{c}89.384 \\
(161.441)\end{array}$ & $\begin{array}{c}16.519 \\
(15.528)\end{array}$ & $\begin{array}{c}8.660 \\
(4.652)\end{array}$ \\
\hline & Recursive & $\begin{array}{c}133.294 \\
(304.348)\end{array}$ & $\begin{array}{l}\mathbf{1 5 . 3 2 5} \\
(9.502)\end{array}$ & $\begin{array}{c}7.389 \\
(\mathbf{2 . 9 7 9 )}\end{array}$ \\
\hline & DirRec & $\begin{array}{c}93.603 \\
(214.783) \\
\end{array}$ & $\begin{array}{l}15.522 \\
(\mathbf{8 . 5 6 5 )} \\
\end{array}$ & $\begin{array}{c}\mathbf{6 . 5 4 3} \\
(3.168) \\
\end{array}$ \\
\hline & ARIMA & $\begin{array}{c}247.719 \\
(534.890)\end{array}$ & $\begin{array}{c}45.805 \\
(27.480) \\
\end{array}$ & $\begin{array}{r}17.929 \\
(5.461) \\
\end{array}$ \\
\hline & ETS & $\begin{array}{c}191.400 \\
(441.876)\end{array}$ & $\begin{array}{l}127.020 \\
(88.968)\end{array}$ & $\begin{array}{c}79.103 \\
(41.610)\end{array}$ \\
\hline
\end{tabular}


The boxplots of the computed RMSPE, MAPE, and AAPRPE values for both stations are presented in Figure 4. From this figure, it is clear the machine learning based methods produce more stable results than the traditional ARIMA and ETS methods. In other words, the traditional ARIMA and ETS methods produce higher error values with increasing forecast horizon, while the machine learning based methods produce similar results for all forecast horizons. In addition, Figure 4 supports the findings reported in Tables 2 and 3 that the recursive and direct strategies produce slightly better performance than the DirRec strategy for short and moderate forecast horizons while DirRec strategy produces improved results than others for long term forecast horizon.
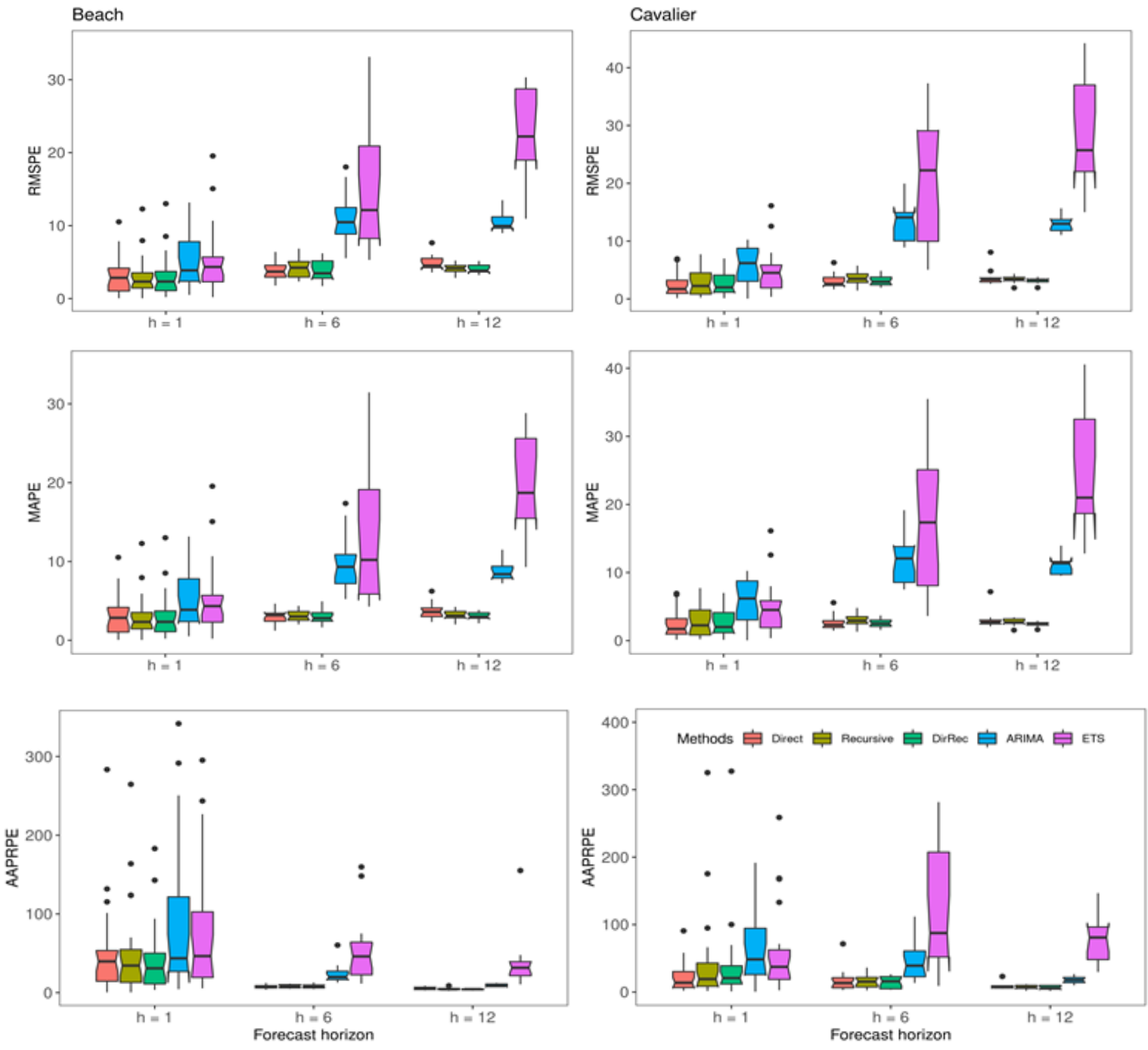

Figure 4. Boxplots of the computed RMSPE, MAPE, and AAPRPE values for $h=1,6$, and 12 steps-ahead forecast horizon. Beach station (first column), Cavalier station (second column).

\section{Discussion}

In this paper, three machine learning based h-steps ahead prediction procedures, i,e., direct, recursive, and DirRec, are discussed and they are applied to two monthly $T_{d e w}$ datasets obtained during 2010-2020 in Beach and Cavalier stations. The predictive performance of these methods are compared with each other's as well as two traditional methods; ARIMA and ETS. The numerical analyses produced from the analyses demonstrate that the machine learning based $h$-steps ahead strategies produce improved forecast results (i.e., smaller forecast errors) compared with traditional methods. When comparing the performance of ARIMA and ETS methods, our results indicate that ARIMA significantly outperforms ETS in most cases. This may be due to fact that the model structures of the methods. While ARIMA is a stationary model, the ETS is non-stationary model. In ARIMA, the dataset is first transformed to a stationary form and then, the forecasts are obtained from the transformed series. Therefore, ARIMA generally produces better forecasts than the ETS. One-step ahead forecast may not be informative in many scientific fields such as hydrology and meteorology because the decision makers in such areas are generally require long-term forecasts to manage risks properly. Thus, 
the presented h-steps ahead forecast strategies in this paper can be used with other hydro-climatic variables such as river flow, drought indices, and rainfall to obtain long-term forecast.

\section{Conclusion}

The current study was established on the forecasting of dew point temperature $\left(T_{\text {dew }}\right)$ using recursive strategy, direct strategy, and DirRec to obtain $h$-steps ahead forecasting. $T_{\text {dew }}$ is considered as highly stochastic and complex hydrological processes and thus its quantification and determination are a challenging task. The modeling development was of Ten years monthly scale dataset of $T_{\text {dew }}$ at two meteorological stations (Beach and Cavalier) located at the North Dakota, USA. The performances of the considered models were validated against ARIMA and ETS models. The proposed methods attained good results for the multi-ahead forecasting.

Conflicts of Interest: The authors declare no conflict of interest.

\section{References}

[1] M. Ali, R. Prasad, Y. Xiang, and Z. M. Yaseen, "Complete ensemble empirical mode decomposition hybridized with random forest and kernel ridge regression model for monthly rainfall forecasts," $J$. Hydrol., 2020.

[2] B. Fersch et al., "High-resolution fully coupled atmospheric-hydrological modeling: a crosscompartment regional water and energy cycle evaluation," Hydrol. Earth Syst. Sci., vol. 24, no. 5, pp. 2457-2481, 2020.

[3] W. Jing et al., "Implementation of evolutionary computing models for reference evapotranspiration modeling: short review, assessment and possible future research directions," Eng. Appl. Comput. Fluid Mech., vol. 13, no. 1, pp. 811-823, 2019.

[4] E. T. Al-Shammari et al., "Prediction of daily dewpoint temperature using a model combining the support vector machine with firefly Algorithm,” J. Irrig. Drain. Eng., vol. 142, no. 5, 2016.

[5] Z. Zhang, S. D. Glaser, R. C. Bales, M. Conklin, R. Rice, and D. G. Marks, "Technical report: The design and evaluation of a basin-scale wireless sensor network for mountain hydrology," Water Resour. Res., 2017.

[6] L. J. Fritschen and P. Doraiswamy, "Dew: An addition to the hydrologic balance of Douglas fir," Water Resour. Res., 1973.

[7] N. F. Attar, K. Khalili, J. Behmanesh, and N. Khanmohammadi, "On the reliability of soft computing methods in the estimation of dew point temperature: The case of arid regions of Iran," Comput. Electron. Agric., vol. 153, pp. 334-346, 2018.

[8] O. Kisi, S. Kim, and J. Shiri, "Estimation of dew point temperature using neuro-fuzzy and neural network techniques," Theor. Appl. Climatol., vol. 114, no. 3-4, pp. 365-373, 2013.

[9] M. Bagheri, S. P. Zhu, M. E. A. Ben Seghier, B. Keshtegar, and N. T. Trung, "Hybrid intelligent method for fuzzy reliability analysis of corroded X100 steel pipelines," Eng. Comput., 2020.

[10] M. Nait Amar, M. A. Ghriga, M. E. A. Ben Seghier, and H. Ouaer, "Prediction of Lattice Constant of A2XY6Cubic Crystals Using Gene Expression Programming," J. Phys. Chem. B, 2020.

[11] S. K. Bhagat, T. Tiyasha, T. M. Tung, R. R. Mostafa, and Z. M. Yaseen, "Manganese (Mn) removal prediction using extreme gradient model," Ecotoxicol. Environ. Saf., vol. 204, p. 111059, Nov. 2020.

[12] A. Sharafati et al., "Development of advanced computer aid model for shear strength of concrete slender beam prediction," Appl. Sci., 2020.

[13] S. Q. Salih, M. Habib, I. Aljarah, H. Faris, and Z. M. Yaseen, "An evolutionary optimized artificial intelligence model for modeling scouring depth of submerged weir," Eng. Appl. Artif. Intell., vol. 96, p. 104012, 2020.

[14] M. Ali, R. C. Deo, Y. Xiang, Y. Li, and Z. M. Yaseen, "Forecasting long-term precipitation for water resource management: a new multi-step data-intelligent modelling approach,” Hydrol. Sci. J., 2020.

[15] M. Mohsenipour, S. Shahid, G. F. Ziarh, and Z. M. Yaseen, "Changes in monsoon rainfall distribution of Bangladesh using quantile regression model," Theor. Appl. Climatol., pp. 1-14, 2020.

[16] S. Maroufpoor, E. Maroufpoor, O. Bozorg-Haddad, J. Shiri, and Z. M. Yaseen, "Soil moisture simulation using hybrid artificial intelligent model: Hybridization of adaptive neuro fuzzy inference system with grey wolf optimizer algorithm," J. Hydrol., 2019.

[17] L. Goel and A. Kaur, "Hybrid Biogeography-Based Optimization Techniques for Geo-Spatial Feature Extraction: A Brief Survey," in Recent Advances on Memetic Algorithms and its Applications in Image Processing, Springer, 2020, pp. 119-152.

[18] M. Bayatvarkeshi, M. A. Imteaz, O. Kisi, M. Zarei, and Z. M. Yaseen, “Application of M5 model tree 
optimized with Excel Solver Platform for water quality parameter estimation,” Environ. Sci. Pollut. Res., pp. 1-18, 2020.

[19] S. K. Bhagat, T. Tiyasha, S. M. Awadh, T. M. Tung, A. H. Jawad, and Z. M. Yaseen, "Prediction of sediment heavy metal at the Australian Bays using newly developed hybrid artificial intelligence models," Environ. Pollut., p. 115663, Sep. 2020.

[20] S. Kim, V. P. Singh, C.-J. Lee, and Y. Seo, "Modeling the physical dynamics of daily dew point temperature using soft computing techniques," KSCE J. Civ. Eng., vol. 19, no. 6, pp. 1930-1940, 2015.

[21] S. Mehdizadeh, J. Behmanesh, and K. Khalili, "Application of gene expression programming to predict daily dew point temperature,” Appl. Therm. Eng., vol. 112, pp. 1097-1107, 2017.

[22] D. B. Shank, G. Hoogenboom, and R. W. McClendon, "Dewpoint temperature prediction using artificial neural networks," J. Appl. Meteorol. Climatol., vol. 47, no. 6, pp. 1757-1769, 2008.

[23] D. B. Shank, R. W. McClendon, J. Paz, and G. Hoogenboom, "Ensemble artificial neural networks for prediction of dew point temperature," Appl. Artif. Intell., vol. 22, no. 6, pp. 523-542, 2008.

[24] M. Zounemat-Kermani, "Hourly predictive Levenberg-Marquardt ANN and multi linear regression models for predicting of dew point temperature," Meteorol. Atmos. Phys., vol. 117, no. 3-4, pp. 181192, 2012.

[25] J. Shiri, S. Kim, and O. Kisi, "Estimation of daily dew point temperature using genetic programming and neural networks approaches," Hydrol. Res., vol. 45, no. 2, pp. 165-181, 2014.

[26] K. Mohammadi, S. Shamshirband, S. Motamedi, D. Petković, R. Hashim, and M. Gocic, "Extreme learning machine based prediction of daily dew point temperature," Comput. Electron. Agric., vol. 117, pp. 214-225, 2015.

[27] M. D. Williams, S. L. Goodrick, A. Grundstein, and M. Shepherd, "Comparison of dew point temperature estimation methods in Southwestern Georgia,” Phys. Geogr., vol. 36, no. 4, pp. 255-267, 2015.

[28] S. N. Qasem, S. Samadianfard, H. S. Nahand, A. Mosavi, S. Shamshirband, and K. Chau, "Estimating Daily Dew Point Temperature Using Machine Learning Algorithms,” Water, vol. 11, no. 3, p. 582, 2019.

[29] M. Alizamir, S. Kim, O. Kisi, and M. Zounemat-Kermani, "Deep echo state network: a novel machine learning approach to model dew point temperature using meteorological variables," Hydrol. Sci. J., 2020.

[30] M. Alizamir, S. Kim, M. Zounemat-Kermani, S. Heddam, N. W. Kim, and V. P. Singh, "Kernel extreme learning machine: An efficient model for estimating daily dew point temperature using weather data," Water (Switzerland), 2020.

[31] A. Sharafati, A. Tafarojnoruz, D. Motta, and Z. M. Yaseen, "Application of nature-inspired optimization algorithms to ANFIS model to predict wave-induced scour depth around pipelines," $J$. Hydroinformatics, 2020.

[32] H. Tao et al., "A Newly Developed Integrative Bio-Inspired Artificial Intelligence Model for Wind Speed Prediction," IEEE Access, vol. 8, pp. 83347-83358, 2020.

[33] M. Jahandideh-Tehrani, G. Jenkins, and F. Helfer, "A comparison of particle swarm optimization and genetic algorithm for daily rainfall-runoff modelling: a case study for Southeast Queensland, Australia," Optim. Eng., 2020.

[34] M. Y. Chia, Y. F. Huang, C. H. Koo, and K. F. Fung, "Recent advances in evapotranspiration estimation using artificial intelligence approaches with a focus on hybridization techniques-A review," Agronomy. 2020.

[35] W. M. Seyoum and D. Kwon, "Suitability of satellite-based hydro-climate variables and machine learning for streamflow modeling at various scale watersheds," Hydrol. Sci. J., 2020.

[36] R. Xu, N. Chen, Y. Chen, and Z. Chen, "Downscaling and Projection of Multi-CMIP5 Precipitation Using Machine Learning Methods in the Upper Han River Basin," Adv. Meteorol., 2020.

[37] S. Dhakal, Y. Gautam, and A. Bhattarai, "Evaluation of Temperature-Based Empirical Models and Machine Learning Techniques to Estimate Daily Global Solar Radiation at Biratnagar Airport, Nepal," Adv. Meteorol., 2020.

[38] A. Sorjamaa, J. Hao, N. Reyhani, Y. Ji, and A. Lendasse, "Methodology for long-term prediction of time series," Neurocomputing, vol. 70, no. 16-18, pp. 2861-2869, 2007.

[39] S. Ben Taieb, G. Bontempi, A. F. Atiya, and A. Sorjamaa, "A review and comparison of strategies for multi-step ahead time series forecasting based on the NN5 forecasting competition," Expert Syst. Appl., vol. 39, no. 8, pp. 7067-7083, 2012.

[40] S. Ben Taieb and R. J. Hyndman, "A gradient boosting approach to the Kaggle load forecasting competition,” Int. J. Forecast., vol. 30, no. 2, pp. 382-394, 2014. 
[41] A. Sorjamaa and A. Lendasse, "Time series prediction using DirRec strategy.," in Esann, 2006, vol. 6, pp. 143-148.

[42] R. J. Hyndman and Y. Khandakar, “Automatic time series forecasting: the forecast package for R,” $J$. Stat. Softw., vol. 27, no. 1, pp. 1-22, 2008. 